\title{
Cárie dentária no Brasil: declínio, polarização, iniqüidade e exclusão social
}

\author{
Paulo Capel Narvai, ${ }^{1}$ Paulo Frazão, ${ }^{1}$ Angelo Giuseppe Roncalli ${ }^{2}$ \\ e José Leopoldo F. Antunes ${ }^{3}$
}

Como citar Narvai PC, Frazão P, Roncalli AG, Antunes JLF. Cárie dentária no Brasil: declínio, iniqüidade e exclusão social. Rev Panam Salud Publica. 2006;19(6):385-93.

RESUMO Objetivo. Analisar a evolução da experiência de cárie dentária entre escolares brasileiros no período de 1980 a 2003 e determinar a distribuição da cárie e o acesso dessa população ao tratamento da doença.

Método. Foram utilizados dados secundários produzidos no período de 1980 a 2003, empregando o indice dentes permanentes cariados, perdidos e restaurados (CPOD). Os estudos que deram origem aos dados variaram quanto ao tipo de investigação, delineamento, plano amostral e critério diagnóstico para a doença, mas produziram estimativas nacionais consideradas válidas para os valores do indice CPOD, admitindo-se como possível a presente análise de tendência.

Resultados. Os valores de CPOD indicaram um nível alto de cárie dentária nos anos 1980, declinando para um nível moderado nos anos 1990. Em 2003, o valor do CPOD ainda era moderado (2,8). Entre 1980 e 2003, o declínio nos valores do CPOD foi de 61,7\%. A porcentagem de escolares com CPOD igual a zero aumentou de 3,7\% em 1986 para 31,1\% em 2003. Por outro lado, enquanto no segmento menos atingido pela doença (CPOD de 1 a 3), o indice de cuidados aumentou de 26,3\% em 1986 para 34,7\% em 2003, no segmento com CPOD de 4 a 5 o indice de cuidados caiu de 50,2\% em 1986 para 39,3\% em 2003. No segmento com CPOD de 6 ou mais, o indice de cuidados se manteve estável (28.0\%). Aproximadamente 20\% da população passou a concentrar cerca de $60 \%$ da carga de doença.

Conclusão. Um declínio relevante do CPOD foi observado no período do estudo, sendo a hipótese explicativa mais plausível a elevação no acesso a água e creme dental fluorados e as mudanças nos programas de saúde bucal coletiva. A despeito da melhora, a distribuição da cárie ainda é desigual. Os dentes atingidos por cárie passaram a se concentrar numa proporção menor de indivíduos. Ademais, não se alterou a proporção de dentes cariados não tratados. A redução das disparidades socioeconômicas e medidas de saúde pública dirigidas aos grupos mais vulneráveis permanecem como um desafio para todos os que formulam e implementam as políticas públicas no Brasil.

Palavras-chave Creme dental, eqüidade no acesso, fluoração, índice CPOD, Brasil.

1 Universidade de São Paulo, Faculdade de Saúde Pública, São Paulo (SP), Brasil. Enviar correspondência a Paulo Capel Narvai no seguinte endereço: Avenida Dr. Arnaldo 715, CEP 01246-904, São Paulo, SP, Brasil. Fone: +55-11-3066-7782.

2 Universidade Federal do Rio Grande do Norte, Faculdade de Odontologia, Natal (RN), Brasil.

3 Universidade de São Paulo, Faculdade de Odontologia, São Paulo (SP), Brasil.
Na maioria dos países desenvolvidos, a prevalência da cárie apresentou uma tendência de declínio nas três últimas décadas do século $X X$ e no início do século XXI $(1,2)$. Contudo, observamse, no interior desses países, diferenças importantes em termos da prevalência da cárie entre regiões e cidades e entre diferentes grupos populacionais (3-5). Tais diferenças vêm sendo bem descritas por diversos autores e caracterizam significativas desigualdades 
em saúde, que requerem a atenção de autoridades e adequadas intervenções de saúde pública $(6,7)$.

As análises de tendências, considerando, por exemplo, a população total de um determinado país, são de grande valor, pois produzem subsídios úteis ao planejamento e à tomada de decisão em saúde. A comparação das tendências de cárie dentária observadas em cada país permite explorar conexões entre as taxas de ocorrência dessa doença ao longo do tempo e aspectos mais gerais, ligados à industrialização, ao desenvolvimento humano e às políticas nacionais de saúde bucal (8).

O propósito deste estudo foi analisar a evolução da experiência de cárie dentária entre escolares brasileiros no período de 1980 a 2003, além de determinar a distribuição da cárie nesse grupo e o acesso dessa população ao tratamento da doença.

\section{MATERIAIS E MÉTODOS}

O Brasil é uma república federativa dividida em cinco macrorregiões (Norte, Nordeste, Centro-Oeste, Sudeste e Sul) e constituída por 26 estados e pelo Distrito Federal. A população, estimada em 184184264 habitantes em 2005 (9), se distribui por 5567 municípios. Cerca de $85 \%$ dos habitantes vivem em áreas urbanas (variando de mais de $90 \%$ no Sudeste a aproximadamente $70 \%$ no Nordeste). Os indicadores sociais brasileiros permanecem relativamente baixos e o País situa-se nas faixas intermediárias na classificação do Índice de Desenvolvimento Humano (IDH) - 0,775 em 2003 (77 ${ }^{\circ}$ posto no conjunto de 177 países) $(10,11)$.

Neste artigo, foram utilizados dados secundários, produzidos no período de 1980 a 2003, provenientes de estudos que utilizaram como instrumento de medida o número de dentes permanentes cariados, perdidos e restaurados (CPOD) (12). A experiência de cárie em dentes permanentes de escolares brasileiros foi identificada na idadeíndice de 12 anos, de acordo com a recomendação da Organização Mundial da Saúde (OMS) (12).
A revisão de artigos e documentos abrangeu um período mais amplo, com início em 1952. Naquele ano, o governo brasileiro começou, mediante uma política explícita, cuja expressão concreta foram os primeiros programas odontológicos escolares, a intervir na área de saúde bucal (13). Contudo, não foram encontrados dados nacionais anteriores a 1980. No processo de busca foram localizados: um artigo contendo o valor do índice CPOD para o ano de 1980 (14), dois documentos oficiais do Ministério da Saúde do Brasil com dados relativos aos anos de 1986 (15) e 2003 (16) e um relatório técnico de um levantamento de abrangência nacional realizado em 1993 por uma organização não-governamental, com um delineamento cujo plano amostral é compatível com a abordagem feita neste trabalho (17). Os valores do índice CPOD para 1996 foram apurados a partir de um banco de dados disponibilizado pelo Ministério da Saúde na Internet (18), uma vez que não foi possível localizar o relatório técnico do levantamento realizado naquele ano. Em consulta feita ao Ministério da Saúde, fomos informados de que o órgão não publicou o relatório daquela pesquisa.

Os estudos que deram origem aos dados variaram quanto ao tipo de investigação, delineamento, plano amostral e critério de diagnóstico para a doença, mas todos produziram estimativas consideradas válidas para os valores do índice CPOD para o período abordado neste trabalho. Em todos os levantamentos, a coleta de dados foi precedida por treinamento de calibração para os examinadores, obtendo-se concordâncias que permitiram sua participação na etapa de campo. Os princípios éticos relativos a levantamentos dessa natureza foram observados em todos os estudos.

Além da consulta ao artigo e aos relatórios técnicos identificados na revisão de literatura, os bancos de dados de 1986 e 2003 foram utilizados para empreender as análises estatísticas e testar o significado das medidas. Tais bancos de dados foram disponibilizados pelo Ministério da Saúde, instituição responsável pela condução das pesquisas. Com relação aos demais estudos: 1) a estimativa produzida para 1980 não gerou um banco de dados que possibilitasse cálculos estatísticos; 2) o levantamento de 1993 apresentou um viés de amostra pois, embora tenha gerado um valor global válido para o índice CPOD, superestima o componente "O" e subestima o componente " $\mathrm{C}$ ", inviabilizando um aspecto da análise feita no presente trabalho; e, 3) o levantamento realizado em 1996 teve um planejamento amostral que também permitiu gerar um valor CPOD válido em termos nacionais, mas com limitações para aferir as situações regionais, inviabilizando outros aspectos da análise aqui empreendida.

Assim, este trabalho focaliza os estudos de 1986 e 2003. Os valores do índice CPOD obtidos nos demais estudos são empregados apenas para a análise de tendência relativa ao país.

Para melhor situar as condições das regiões, são apresentados dados sociodemográficos com base no ano 2000, a saber: a) porcentagem da população total (169 872856 habitantes) que habita cada região; b) renda per capita (em dólares americanos), c) média de anos de estudo das pessoas de 25 anos de idade ou mais; e d) porcentagem das pessoas que vivem em domicílios com água encanada.

$\mathrm{O}$ acesso da população ao tratamento foi analisado com base na utilização de serviços odontológicos, medida pelo índice de cuidados odontológicos (razão entre dentes restaurados e o total de dentes com experiência de cárie) (19).

Também o fenômeno epidemiológico da polarização da cárie foi analisado, com base nos estudos de 1986 e 2003. Foi empregada a curva de Lorenz para avaliar as características da distribuição da cárie nesse intervalo temporal $(20,21)$. O termo "polarização" foi definido como concentração de $75 \%$ das cáries em 25\% das crianças (22).

Além da análise na população como um todo, os valores de CPOD dos escolares de cada uma das cinco regiões brasileiras também foram examinados, apurando-se as diferenças relativas e comparando-se as mudanças ocorridas entre a melhor e a pior região. 
FIGURA 1. Índice CPOD segundo o ano para a idade-índice de 12 anos, Brasil

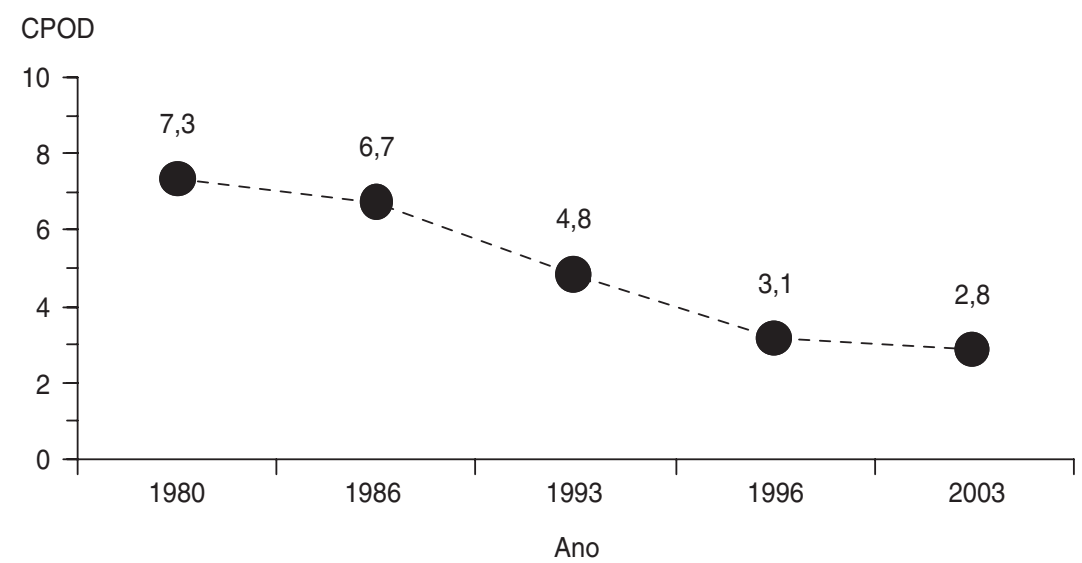

Fonte: Pinto (14), Brasil (15-17), SESI (18). dessas regiões e as regiões Sudeste e Sul $(P=0,000)$. Estas, por sua vez, não diferem entre si $(P=0,821)$. Comparando-se as diferenças relativas nos valores de CPOD entre as regiões CentroOeste (pior) e Sudeste (melhor) em 1986 $(8,5 / 5,9)$ e em $2003(3,2 / 2,3)$, pode-se observar que a disparidade interregional foi mantida em cerca de 1,4.

Na tabela 1 são apresentados os valores médios do CPOD e do coeficiente de Gini para 1986 e 2002, por região, bem como o IDH, a renda per capita, a escolaridade e a porcentagem das pessoas com acesso à água encanada em 2000.

A figura 3 mostra a composição percentual do índice CPOD para os estudos realizados em 1986 e 2003. Destaca-se a semelhança no perfil dessa composição, com os componentes registrando, respectivamente: $\mathrm{C}=$ $61,6 \%$ e $60,8 \%(P=0,520), P=6,6 \%$ e $6,5 \%(P=0,861)$ e $\mathrm{O}=31,8 \%$ e $32,7 \%$ $(P=0,418)$. Cabe assinalar, contudo, que no segmento menos atingido pela doença (CPOD de 1 a 3), o índice de cuidados aumentou de 26,3\% em 1986 para $34,7 \%$ em 2003. No segmento em que os valores do CPOD variaram de 4 a 5, o índice de cuidados caiu de $50,2 \%$ em 1986 para $39,3 \%$ em 2003 . No segmento severamente atingido pela doença (CPOD de 6 ou mais), o índice de cuidados se manteve estável (28,0\% em 1986, e 28,1\% em 2003).

Na figura 4 encontram-se as distribuições percentuais dos valores do índice CPOD em 1986 e em 2003, evidenciando uma tendência à concentração da cárie entre os escolares brasileiros. Observa-se que no pólo da ausência de doença, cujo indicador é o $\mathrm{CPOD}=0$, estão apenas 3,7\% dos escolares em 1986. Essa porcentagem aumenta para $31,1 \%$ em 2003 . No outro pólo, onde se concentra o maior número de dentes CPO (4 ou mais), estão $80,5 \%$ em 1986 e 33,5\% em 2003. A diminuição nos níveis populacionais de cárie é acompanhada por expressiva redução na magnitude da ocorrência, com uma porcentagem muito maior de indivíduos com CPOD na faixa de 1 a 3 dentes atingidos em 2003 em relação a 1986 (35,4 e 15,8\%, respectivamente).

Essa tendência de concentração é mostrada na figura 5, que compara a 
TABELA 1. Índice CPOD e coeficiente de Gini em 1986 e 2002 e indicadores sociodemográficos em 2000 para as regiões brasileiras

\begin{tabular}{|c|c|c|c|c|c|c|}
\hline \multirow[b]{2}{*}{ Índice } & \multicolumn{6}{|c|}{ Região } \\
\hline & Norte & Nordeste & Sudeste & Sul & Centro-Oeste & Brasil \\
\hline \multicolumn{7}{|l|}{ CPOD } \\
\hline 1986 & 7,50 & 6,90 & 5,95 & 6,31 & 8,53 & 6,65 \\
\hline 2003 & 3,13 & 3,18 & 2,30 & 2,31 & 3,16 & 2,79 \\
\hline \multicolumn{7}{|l|}{ Gini } \\
\hline 1986 & 0,275 & 0,366 & 0,342 & 0,359 & 0,279 & 0,341 \\
\hline 2003 & 0,523 & 0,565 & 0,603 & 0,600 & 0,542 & 0,572 \\
\hline \multicolumn{7}{|l|}{ Porcentagem } \\
\hline da população & 7,60 & 28,13 & 42,64 & 14,78 & 6,85 & 100,00 \\
\hline $\mathrm{IDH}^{\mathrm{b}}$ & 0,664 & 0,610 & 0,745 & 0,771 & 0,737 & 0,699 \\
\hline Renda $^{c}$ & 66,07 & 46,71 & 121,89 & 128,16 & 114,71 & 93,68 \\
\hline Escolaridade $^{d}$ & 3,53 & 2,91 & 4,70 & 4,88 & 4,42 & 4,04 \\
\hline$\%$ de água encanada & 34,7 & 41,2 & 88,8 & 91,8 & 78,6 & 68,7 \\
\hline
\end{tabular}

\footnotetext{
a Porcentagem relativa a cada região considerando a população total de 169799170 habitantes em 2000

b Índice de desenvolvimento humano em 2000.

c Renda per capita em dólares americanos em 2000.

d Média de anos de estudo das pessoas de 25 anos de idade ou mais em 2000.

e Porcentagem das pessoas que viviam em domicílios com água encanada em 2000.
}

distribuição dos valores em 1986 e em 2003, com uma distribuição uniforme esperada no caso de completa igualdade. Concomitante à redução da experiência de cárie, observa-se um aumento da desigualdade entre os valores de CPOD aos 12 anos de idade para o país.

Cada ponto de cruzamento dos eixos X e Y, em 1986 e em 2003, indica, cumulativamente, a proporção de indivíduos (eixo $\mathrm{X}$ ) com cárie (eixo Y), ambos os eixos variando de 0 a 1 (ou 0 a 100\%). Constata-se que, no extremo à esquerda do eixo $X$, a distribuição de $20 \%$ da carga da doença mudou de cerca de $40 \%$ para aproximadamente $60 \%$ da população. É o pólo dos menos atingidos. No extremo oposto, no lado
FIGURA 3. Composição percentual do índice CPOD aos 12 anos de idade, Brasil, 1986 e 2003

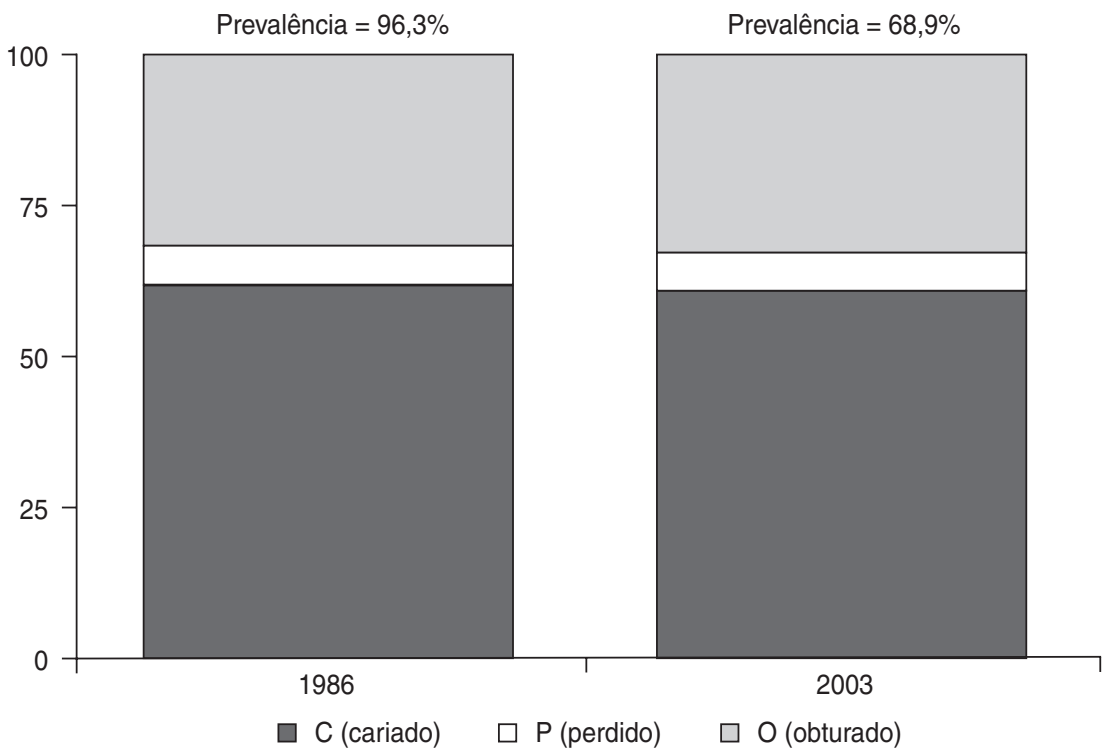

direito do eixo $\mathrm{X}$, no pólo dos mais atingidos, aproximadamente $20 \%$ da população passou a concentrar cerca de $60 \%$ da carga de doença.

\section{DISCUSSÃO}

\section{Tendências da cárie no Brasil e fatores associados}

Entre 1980 e 2003, a redução nos valores do índice CPOD mostra uma tendência consistente de queda ao longo do período, que corresponde a um declínio de $61,7 \%$. Tal redução é observada para o país como um todo e para as cinco regiões, evidenciando que todas contribuem para o declínio nacional do CPOD.

O declínio na prevalência e na severidade da cárie dentária tem sido relatado nos diferentes países americanos. Beltrán-Aguilar et al. (23) analisaram dados relativos às Américas, abrangendo o período de 1970 a 1990, e concluíram que houve declínio no período. Bönecker e Cleaton-Jones (24), enfocando a situação da cárie na América Latina e no Caribe nas três últimas décadas do século $X X$, relataram uma tendência de diminuição na prevalência e severidade da doença, com menor força de declínio nos últimos 10 anos.

A comparação de estimativas geradas por diferentes estudos pode não ser apropriada em razão das características metodológicas de cada investigação (25). A falta de padronização na aferição dos eventos e a obtenção de amostras não-representativas da população de referência, entre outros aspectos, podem comprometer significativamente as estimativas geradas e, conseqüentemente, a comparação dos resultados. Alguns autores $(25,26)$ afirmaram que as mudanças nos critérios para identificar a cárie podem contribuir para explicar o declínio observado na sua prevalência. Comparando-se os critérios de padronização empregados nos principais levantamentos documentados no Brasil, podem ser observadas diferenças na função básica dos instrumentos. A importância da dimensão táctil do exame, forte no passado, vem cedendo lugar para a di- 
FIGURA 4. Distribuição percentual dos valores do índice CPOD aos 12 anos de idade, Brasil, 1986 e 2003

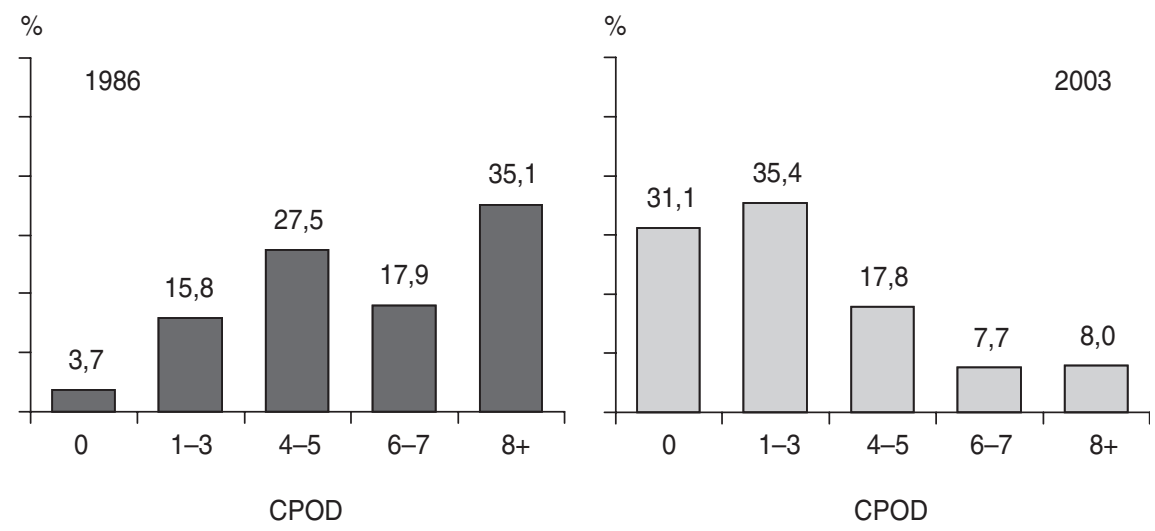

FIGURA 5. Porcentagem acumulada de dentes cariados em função da porcentagem acumulada de indivíduos de 12 anos de idade, Brasil, 1986 e 2003

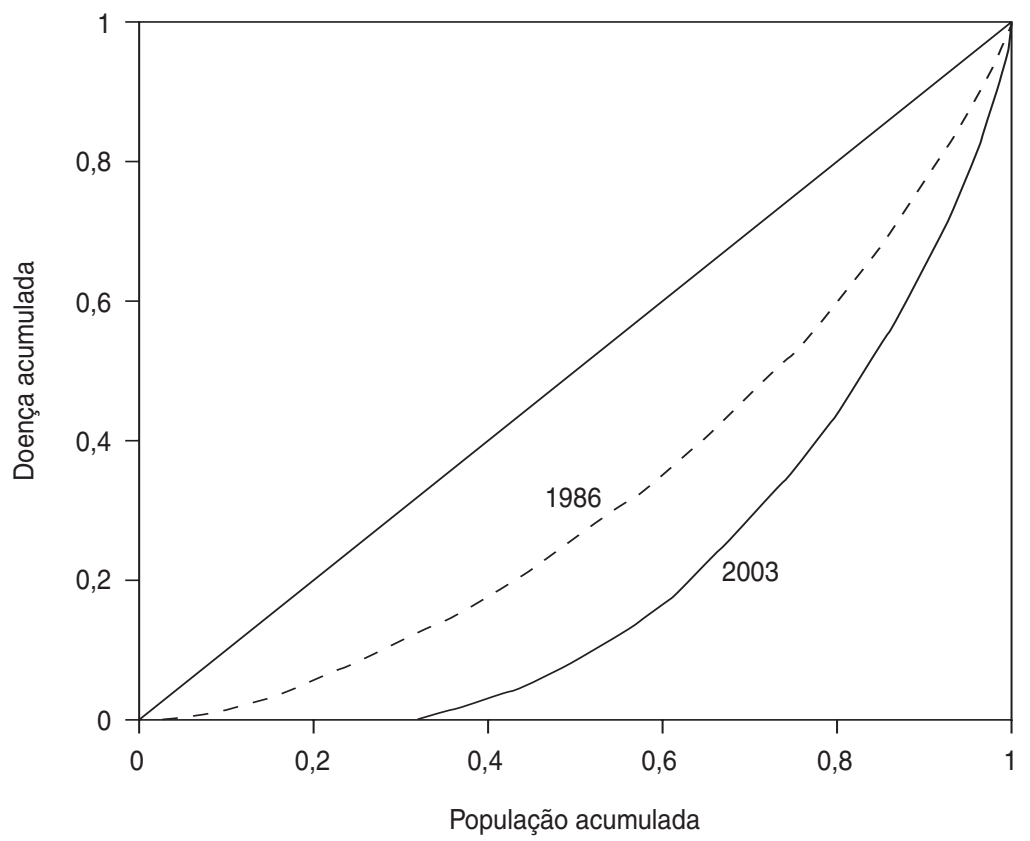

mensão visual, na qual a sonda é empregada somente para remover depósitos sobre a superfície examinada, identificar selantes, checar alterações visualmente detectadas e avaliar, suavemente, a textura de superfícies e tecidos, incluindo a presença de amolecimento em bases ou paredes de lesões, as quais não se deve mais pressionar. Manchas brancas ou escuras, rugosidades e descolorações sem evidência de cavitação ou esmalte socavado (sem suporte) não têm sido condições de registro de dentes cariados nos levantamentos analisados. Assim, as modificações relatadas no índice CPOD, embora impliquem cuidados na interpretação e comparação dos dados (2527), não alteraram as estimativas produzidas (28) a ponto de invalidar análises de tendência geral como a que se apresenta neste artigo.
Conforme mencionado, as técnicas de amostragem de cada estudo não foram semelhantes. Por essa razão, o foco da análise foi centrado nas estimativas produzidas em 1986 e 2003.

No levantamento da população escolar, realizado em 1986, foram sorteadas 105 escolas de ensino fundamental e 24 escolas de ensino secundário, abrangendo a zona urbana de 16 capitais brasileiras, numa fração de amostragem que variou de $1 / 200$ a $1 / 500$, conforme a região do país. Em cada unidade escolar foram sorteados cerca de 15 escolares de 12 anos, para um total de 1792 registros.

No levantamento de 2003, o processo de amostragem por conglomerados envolveu 250 municípios distribuídos em cinco tipos de porte $(<5000$ habitantes, 5000 a 9 999, 10000 a 49 999, 50000 a 99999 e $\geq 100000$ habitantes) nas cinco regiões. Foram incluídas milhares de escolas urbanas e rurais dispersas por todo o território, inclusive 50 cidades na região amazônica, área de difícil acesso. Foram obtidos 34550 registros (29).

Dadas as características para a obtenção de uma amostra probabilística em ambos os estudos, foi assumido igual peso para todos os elementos amostrais. Embora somente escolas urbanas de 16 cidades tenham participado das unidades amostrais em 1986, é preciso considerar que, naquela época, a distribuição da cárie era mais homogênea na população. Por esses aspectos, admite-se que a redução observada foi gerada por estimativas válidas, sendo esses dados a mais acurada informação disponível sobre escolares brasileiros na época.

A redução dos valores do CPOD tem sido atribuída à expansão da fluoração das águas de abastecimento público (sobretudo no Sul e Sudeste), à introdução de dentifrícios fluorados no mercado (com maior impacto relativo nas regiões Norte, Nordeste e Centro-Oeste) e à mudança de enfoque nos programas de odontologia em saúde pública em todas as regiões do país (30). A efetividade da fluoração das águas no declínio da cárie é um fato amplamente aceito, tanto por especialistas em saúde pública quanto 
pela comunidade odontológica, no Brasil e em nível internacional $(29,31)$. É reconhecida tanto a efetividade da ação na localidade fluorada quanto em outras áreas, dado o efeito "halo", mencionado por Griffin et al. (32), segundo o qual a fluoração da água beneficia indiretamente outras comunidades, uma vez que o flúor é também veiculado nos alimentos e bebidas produzidos nas localidades fluoradas. No Brasil, em razão, entre outros aspectos, da grande variabilidade ambiental entre as regiões e dos diferentes teores de flúor naturalmente existentes na água, não se adiciona flúor ao sal de cozinha nem ao leite (33).

A cobertura da fluoração expandiuse notavelmente em todo o país na segunda metade do século $\mathrm{XX}$, notadamente nos anos 1980, em decorrência da decisão governamental federal de apoiar financeiramente iniciativas nessa área, atingindo aproximadamente 65,5 milhões (cerca de $42,2 \%$ da população) de pessoas em 1995. É relevante registrar que, em 1972, apenas 3,3 milhões de brasileiros tinham acesso à água fluorada (33).

Em setembro de 1988, o fabricante do dentifrício líder em vendas em todo o país introduziu flúor no produto e, a partir de 1989, estavam fluorados mais de $90 \%$ dos produtos disponíveis para os consumidores (34). Em 1981, apenas cerca de $12 \%$ dos produtos consumidos no Brasil eram fluorados (35). Atualmente, contudo, praticamente todos os dentifrícios comercializados no país contêm fluoretos. Segundo os produtores brasileiros, em 1997 os brasileiros consumiam $508 \mathrm{~g}$ de dentifrício per capita-um dos melhores índices em nível mundial, com tendência de aumento (30). Os produtos importados corresponderam, em 1997, a 1,2\% do mercado, evidenciando uma situação de não-dependência e de preços não onerados por taxas de importação.

Ainda que se saiba que é reduzida a contribuição dos serviços odontológicos na alteração dos níveis de cárie dentária em populações (36), é prudente considerar que, no caso brasileiro, centenas de programas de odontologia em saúde pública foram alterados nas três últimas décadas. De- dicados basicamente às restaurações e extrações dentárias em escolares de ensino fundamental, vários programas, bem organizados e dirigidos em nível municipal (em decorrência da descentralização do sistema de saúde brasileiro), foram modificados, passando a utilizar o referencial da odontologia preventiva e da promoção da saúde bucal. Em nível nacional, essa mudança programática foi especialmente impulsionada pelo governo federal, que, a partir de 1991, definiu financiamento específico para a realização de procedimentos coletivos em saúde bucal, criando condições favoráveis à expansão desses sistemas de prevenção em unidades escolares e outros espaços sociais (30). Outras hipóteses que poderiam ser consideradas para explicar o declínio observado envolvem variáveis reconhecidas como capazes de influenciar os níveis de prevalência e a magnitude da cárie dentária em populações e dizem respeito ao padrão de consumo de produtos açucarados, à distribuição da renda nacional e à escolaridade dos pais, sobretudo da mãe. Não se registraram, entretanto, para o período analisado, alterações positivas significativas nessas variáveis, cuja dimensão possa explicar o declínio.

Com efeito, o número médio de anos de estudo dos responsáveis pelos domicílios no Brasil era 4,8 em 1991 (4,9 para os homens e 4,8 para as mulheres). Em 2000, essa média se elevou para 5,7 (5,7 para os homens e 5,6 para as mulheres), com grande variabilidade regional $(7,9$ anos para as mulheres do Distrito Federal contra 3,8 para as do Piauí) $(37,38)$. Embora esse aumento de cerca de 1 ano seja relativamente muito expressivo, considerando os níveis médios, há que admitir que é muito pequeno o poder explicativo dessa variável no declínio, tendo em vista que a média de anos de estudo é muito baixa. Ademais, cerca de $20 \%$ das mulheres brasileiras de 20 anos ou mais de idade não são alfabetizadas (38).

O Brasil é o maior produtor mundial de açúcar e o produto, sob diferentes formas, é fartamente consumido, sem que mudanças significativas nos padrões de consumo tenham sido re- gistradas. É certo que, no período de 1986 a 2003, nada de relevante aconteceu com essa variável que possa explicar o declínio nos valores do índice CPOD. Em estudo sobre a disponibilidade de macronutrientes em áreas metropolitanas brasileiras, Monteiro et al. (39) constataram que, entre 1988 e 1996, foi mínima a diferença $(1,2 \%)$ nas porcentagens relativas à disponibilidade de sacarose. Pode-se afirmar que um aumento de 23,2 para $24,4 \%$ não tem qualquer significado em termos de cárie dentária, não explicando diferenças populacionais. Ainda, nada indica que, nesse aspecto, o que aconteceu nas áreas metropolitanas difira significativamente das demais áreas do País.

Quanto à distribuição da renda nacional, constata-se a manutenção, no período do estudo, de um perfil extremamente desfavorável à promoção da saúde, com extrema concentração: o índice de Gini se mantém em torno de 0,6 , em um cenário onde os $50 \%$ mais pobres ficam com cerca de $12 \%$ da riqueza produzida no país, enquanto os $1 \%$ mais ricos ficam com aproximadamente $13 \%$ (40). A participação das rendas do trabalho na renda interna total do Brasil piorou: era de $48 \%$ em 1989 e diminuiu para 40\% em 2003. Em conseqüência, aumentou a porcentagem das rendas do capital: de 52\% para $60 \%$, respectivamente $(40)$. A manutenção desse perfil permite afirmar que a renda e sua distribuição têm escasso poder explicativo para o declínio nos valores do índice CPOD no Brasil.

\section{Características da polarização no Brasil}

Embora não haja concordância entre os diferentes autores $(21,22,41,42)$ ao definir mais precisamente o fenômeno da "polarização", todos compartilham a noção geral de que há polarização quando num pólo há ausência de doença e, no outro, um grande número de casos concentrados num pequeno grupo de indivíduos. Para Dimitrova et al. (42) a polarização implica tãosomente uma "grande porcentagem de indivíduos livres de cárie". 
Examinando o processo de transição epidemiológica da cárie dentária na população infantil nos países mais desenvolvidos, observa-se declínio com a polarização. Os valores cada vez mais baixos de CPOD aos 12 anos de idade encontrados indicam redistribuição de uma menor carga de doença. Além disso, cada vez mais a distribuição da cárie vai se afastando de uma distribuição uniforme, sendo notados níveis crescentes de desigualdade. A polarização é um fenômeno que pode estar refletindo medidas de prevenção e controle da enfermidade embasadas na estratégia populacional (43). Evolui-se de uma situação de alta prevalência para um cenário em que se constata uma grande porcentagem de indivíduos livres de cárie.

No Brasil, $20 \%$ da população de escolares passou a concentrar cerca de $60 \%$ da carga de doença; a diferença relativa entre as regiões não foi alterada. Esse quadro pode estar expressando um outro fenômeno: o da iniquiidade, em que, no caso da cárie, o ataque desigual da doença entre os indivíduos decorre não apenas de variações biológicas inevitáveis, mas também das diferenças que têm origem na ordem social onde estão inseridos, e que se expressam por meio do processo saúde-doença. Ao analisar indicadores de saúde no Brasil, Almeida et al. (44) afirmaram que, embora a expectativa de vida tenha aumentado e os valores dos coeficientes de mortalidade geral, e por causas específicas, tenham diminuído nas últimas décadas, persistem importantes diferenças entre as regiões e variações segundo o nível de renda.

\section{Declínio do CPOD e aumento das desigualdades}

Os conceitos de igualdade, desigualdade, equidade e iniqüidade vêm sendo utilizados no período mais recente, também nos estudos epidemiológicos de cárie dentária, com a finalidade de compreender melhor os padrões de distribuição populacional da doença. Desigualdade e igualdade são conceitos relativos a dimensões e dizem respeito a quantidades mensuráveis. Em sentido distinto, iniqüidade e eqüidade são conceitos políticos que expressam compromisso moral com a justiça social. Nem toda a desigualdade se reveste necessariamente de um caráter injusto. De modo geral, as diferenças resultantes de variação biológica e de comportamentos perigosos à saúde livremente escolhidos, tais como participação em certos esportes, não são normalmente consideradas como iniqüidade (45).

No contexto brasileiro, apesar dos inegáveis avanços no declínio do CPOD, ainda persiste um quadro de iniqüidade na distribuição da cárie, que pode ser explicado pelas precárias condições de existência a que é submetida a ampla maioria da população, configurando um quadro já descrito como de apartheid social (46).

A composição percentual do índice CPOD para 1986 e 2003 (figura 3) mostra que, embora tenha ocorrido um expressivo declínio na experiência de cárie entre os escolares brasileiros, permanece inalterado o padrão dessa composição. $\mathrm{O}$ fato de ter dobrado a proporção de dentistas por 10 mil habitantes de 1980 a 2003 (de cerca de 5 para aproximadamente 10) (47) parece não ter sido capaz de alterar significativamente esse padrão de exclusão do acesso aos cuidados odontológicos, que permanece a desafiar a sociedade e as autoridades públicas. É pertinente, a respeito, a distinção entre os conceitos de eqüidade em saúde e eqüidade no uso de serviços de saúde. Os determinantes das desigualdades em saúde não são os mesmos das desigualdades no consumo de serviços de saúde, como também a eqüidade no uso de serviços de saúde não resulta, necessariamente, em eqüidade na situação de saúde (48).

A ampliação da cobertura dos serviços odontológicos, decorrente da descentralização do sistema de saúde brasileiro e dos investimentos no setor, assim como da maior disponibilidade de recursos odontológicos privados para a sociedade, não foi suficiente para alterar significativamente o padrão de composição do índice CPOD. Observase que a participação do componente " $\mathrm{C}$ " (dentes cariados, incluindo aqueles com extração indicada) continua sendo extremamente elevada, respondendo por aproximadamente dois terços do valor total do índice CPOD, com 64,7\% em 1980 e 60,8\% em 2003. O predomínio do componente " $\mathrm{C}$ " na composição do CPOD evidencia que, a despeito da diminuição na prevalência e magnitude da cárie entre escolares brasileiros, o acesso aos serviços para restaurar dentes continua sendo um enorme desafio para o Estado e a sociedade no Brasil, aprofundando a contradição de um país onde não faltam dentistas, mas onde a população não consegue fazer valer o seu direito de acesso aos cuidados proporcionados por esses profissionais. O sistema público de saúde responde por apenas $24 \%$ dos atendimentos odontológicos-e por $52 \%$ dos atendimentos não-odontológicos (49).

Tal característica conforma um quadro em que o declínio na prevalência da doença não vem sendo acompanhado nem pela modificação dos padrões assistenciais, nem pela inversão dos componentes do índice CPOD. Por essa razão, pode-se denominar de iníquo o declínio dos valores do CPOD. À diminuição da magnitude de ocorrência da cárie não têm correspondido mudanças nos padrões de acesso aos serviços, nem ao tipo de cuidado ofertado aos escolares. As restaurações dentárias correspondem a cerca de um terço dos cuidados ofertados, e as exodontias, expressão cruel da mutilação dentária precoce, mantémse com porcentagens superiores a $6 \%$ dos dentes afetados por cárie. Dados disponibilizados pelo Ministério da Saúde mostram que, no serviço público, as extrações dentárias correspondem a $14 \%$ de todos os procedimentos básicos ofertados, sendo que essa proporção varia de $22 \%$ no Nordeste a $8 \%$ no Sudeste. Em alguns municípios, a proporção chega a 100\%, ou seja, o único procedimento de atenção básica disponível nessas localidades é a extração dentária. Embora a Constituição brasileira afirme a saúde como direito de todos e dever do Estado, sendo a universalidade do acesso às ações e aos serviços de saúde para a sua promoção, proteção e recuperação um dos princípios constitucionais do 
sistema de saúde do país (50), isso não vem se concretizando no que diz respeito aos serviços odontológicos.

Iniqüidades e mudanças epidemiológicas na saúde materno-infantil brasileira têm sido avaliadas (51). As tendências da cárie mostradas neste estudo corroboram tais análises. A elevação do acesso tanto à água quanto ao creme dental fluorados têm resultado em expressiva diminuição na prevalência geral da cárie dentária. Contudo, tais estratégias não têm sido suficientes para reduzir as desigualdades entre as regiões e na população escolar como um todo.

Medidas sociais e econômicas mais gerais, voltadas ao enfrentamento da exclusão social e intervenções de saúde pública complementares, dirigidas aos grupos mais vulneráveis, tanto no campo da promoção da saúde bucal quanto no âmbito mais restrito da assistência odontológica, continuam a desafiar os formuladores e gestores de políticas públicas brasileiras. Nesse sentido, a $3^{a}$ Conferência Nacional de Saúde Bucal (CNSB), realizada em 2004 (52), apresentou 298 propostas que incluem as dimensões da educação e construção da cidadania, controle social e gestão participativa, formação e trabalho em saúde bucal e financiamento e organização da atenção. Tais propostas foram aprovadas por 883 delegados, eleitos em 27 conferências estaduais, das quais, por sua vez, participaram representantes indicados em 2542 conferências municipais ou regionais de saúde bucal. A CNSB foi um processo que possibilitou a ampla participação de agentes sociais representativos de diferentes segmentos populacionais na formulação e apresentação de propostas sobre saúde bucal às autoridades públicas, com o envolvimento de cerca de 90000 pessoas em todo o país. Esperase que, com esse movimento, seja possível manter o declínio observado no índice CPOD e, ainda que convivendo com um certo grau de desigualdade na distribuição da doença, eliminar o caráter iníquo dessa desigualdade.

\section{REFERÊNCIAS}

1. World Health Organization. The World Oral Health Report 2003. Continuous improvement of oral health in the 21st century-the approach of the WHO Global Oral Health Programme. Genebra: WHO; 2003.

2. Nithila A, Bourgeois D, Barmes DE, Murtomaa H. WHO Global Oral Data Bank, 198696: an overview of oral health surveys at 12 years of age. Bull World Health Org. 1998; 76(3):237-44.

3. Jones CM, Taylor GO, Whittle JG, Evans D, Trotter DP. Water fluoridation, tooth decay in 5 year olds, and social deprivation measured by the Jarman score: analysis of data from British dental surveys. BMJ. 1997;315(7107): 514-7.

4. Vargas CM, Crall JJ, Schneider DA. Sociodemographic distribution of pediatric dental caries: NHANES III, 1988-1994. J Am Dent Assoc. 1998;129(9):1229-38.

5. Antunes JLF, Narvai PC, Nugent ZJ. Measuring inequalities in the distribution of dental caries. Community Dent Oral Epidemiol. 2004;32(1):41-8.

6. Sheiham A. Changing trends in dental caries. Int J Epidemiol. 1984;13(2):142-7.

7. Pattussi MP, Marcenes W, Croucher R, Sheiham A. Social deprivation, income inequality, social cohesion and dental caries in Brazilian school children. Soc Sci Med. 2001; 53(7):915-25.

8. Lalloo R, Myburgh NG, Hobdell MH. Dental caries, socio-economic development and national oral health policies. Int Dent J. 1999; 49(4):196-202.

9. Instituto Brasileiro de Geografia e Estatística (IBGE), Diretoria de Pesquisas, Coordenação de População e Indicadores Sociais, Gerência de Estudos e Análises da Dinâmica Demográfica. Projeção da população do Brasil por sexo e idade para o período 1980-2050. Revisão 2004. Disponível em: www.ibge.gov.br. Acessado em 18 de fevereiro de 2005.
10. Instituto de Pesquisa Econômica Aplicada. Report on human development in Brazil. Brasília: IPEA; 2001.

11. Programa das Nações Unidas para o Desenvolvimento. Relatório de Desenvolvimento Humano 2004: liberdade cultural num mundo diversificado. Nova Iorque: PNUD; 2004.

12. World Health Organization. Oral health surveys: basic methods. 4th ed. Genebra: WHO; 1997.

13. Narvai PC. Odontologia e saúde bucal coletiva. $2^{a}$ ed. São Paulo: Ed. Santos; 2002.

14. Pinto VG. Saúde bucal no Brasil. Rev Saude Publ. 1983;17:316-27.

15. Brasil, Ministério da Saúde, Secretaria Nacional de Programas Especiais de Saúde, Divisão Nacional de Saúde Bucal, Fundação Serviços de Saúde Pública. Levantamento epidemiológico em saúde bucal: Brasil, zona urbana, 1986. Brasília: CD-MS; 1988.

16. Brasil, Ministério da Saúde, Secretaria de Atenção à Saúde, Departamento de Atenção Básica, Coordenação Nacional de Saúde Bucal. Projeto SB Brasil 2003-Condições de saúde bucal da população brasileira 2002-2003: resultados principais. Brasília: MS-CNSB; 2004.

17. Serviço Social da Indústria. Estudo epidemiológico sobre prevalência da cárie dental em crianças de 3 a 14 anos: Brasil, 1993. Brasília: SESI-DN; 1996.

18. Brasil, Ministério da Saúde, Secretaria de Políticas de Saúde, Área Técnica de Saúde Bucal. Levantamento epidemiológico de cárie dentária, 1996-banco de dados. Disponível em: www.saude.gov.br. Acessado em 15 de maio de 2006.

19. Walsh J. International patterns of oral health care - the example of New Zealand. N Z Dent J. 1970;66(304):143-52.

20. Measuring health inequalities: Gini coefficient and concentration index. Epidemiol Bull. 2001;22(1):3-4.
21. Tickle M. The 80:20 phenomenon: help or hindrance to planning caries prevention programmes? Community Dent Health. 2002; 19(1):39-42.

22. Macek MD, Heller KE, Selwitz RH, Manz MC. Is 75 percent of dental caries really found in 25 percent of the population? J Public Health Dent. 2004;64(1):20-5.

23. Beltrán-Aguilar ED, Estupiñán-Day S, Baez R. Analysis of prevalence and trends of dental caries in the Americas between the 1970s and 1990s. Int Dent J. 1999;49(6):322-9.

24. Bönecker M, Cleaton-Jones P. Trends in dental caries in Latin American and Caribbean 5-6- and 11-13-year-old children: a systematic review. Community Dent Oral Epidemiol. 2003;31(2):152-7.

25. Warpeha R, Beltrán-Aguilar E, Baez R. Methodological and biological factors explaining the reduction in dental caries in Jamaican school children between 1984 and 1995. Rev Panam Salud Publica. 2001;10(1):37-44.

26. Marcenes W, Freysleben GR, Peres MA. Contribution of changing diagnostic criteria toward reduction of caries between 1971 and 1997 in children attending the same school in Florianópolis, Brazil. Community Dent Oral Epidemiol. 2001;29(6):449-55.

27. Burt BA, Eklund SA. Measuring dental caries. Em: Burt BA. Dentistry, dental practice, and the community. $5^{\mathrm{a}}$ ed. Filadélfia: WB Saunders; 1999. Pp. 178-84.

28. Narvai PC, Biazevic MGH, Junqueira SR, Pontes ERCJ. Diagnóstico de cárie dentária: comparação dos resultados de três levantamentos epidemiológicos numa mesma população. Rev Bras Epidemiol. 2001;4(2):72-80.

29. Antunes JL, Peres MA, de Campos de Mello TR, Waldman EA. Multilevel assessment of determinants of dental caries experience in Brazil. Community Dent Oral Epidemiol. 2006;34(2):146-52. 
30. Narvai PC, Frazão P, Castellanos RA. Declínio na experiência de cárie em dentes permanentes de escolares brasileiros no final do século XX. Odont Soc. 1999;1(1/2):25-9.

31. Recommendations for using fluoride to prevent and control dental caries in the United States. Centers for Disease Control and Prevention. MMWR Recomm Rep. 2001;50(RR14):1-42.

32. Griffin SO, Gooch BF, Lockwood SA, Tomar SL. Quantifying the diffused benefit from water fluoridation in the United States. Community Dent Oral Epidemiol. 2001;29(2): 120-9.

33. Narvai PC. Cárie dentária e flúor: uma relação do século XX. Cienc Saude Coletiva. 2000;5(2): 381-92.

34. Cury JA, Tabchoury CPM. Determination of appropriate exposure to fluoride in non-EME countries in the future. J Appl Oral Sci. 2003; 11(2):83-95.

35. Bastos JRM, Lopes ES. Dentifrícios: cosméticos e terapêuticos. Bauru: FOB/Universidade de São Paulo; 1984. (Série Publicações Científicas $\mathrm{n}^{\circ} 001 / 84$ ).

36. Nadanovsky P, Sheiham A. Relative contribution of dental services to the changes in caries levels of 12-year-old children in 18 industrialized countries in the 1970s and early 1980s. Community Dent Oral Epidemiol. 1995;23(6): 331-9.

37. Instituto Brasileiro de Geografia e Estatística. Censo Demográfico 1991 e 2000. Disponível em: www.ibge.gov.br. Acessado em 18 de fevereiro de 2005.

38. Instituto Brasileiro de Geografia e Estatística, Diretoria de Pesquisas. Perfil das mulheres responsáveis pelos domicílios no Brasil2000. Rio de Janeiro: IBGE; 2002. (Estudos e Pesquisas $n^{\circ} 8$ )

39. Monteiro CA, Mondini L, Costa RBL. Mudanças na composição e adequação nutricional da dieta familiar nas áreas metropolitanas do Brasil (1988-1996). Rev Saude Publ. 2000; 34(3):251-8.

40. Instituto Brasileiro de Geografia e Estatística (IBGE), Diretoria de Pesquisas. Indicadores sócio-econômicos. Disponível em: www.ibge. gov.br. Acessado em 18 de fevereiro de 2005.

41. Vehkalahti M, Tarkkonen L, Varsio S, Heikkila P. Decreasing and polarization of dental caries occurrence among child and youth populations, 1976-1993. Caries Res. 1997;31(3): 161-5.

42. Dimitrova MM, Kukleva MP, Kondeva VK. A study of caries polarization in 1-, 2- and 3year-old children. Folia Med (Plovdiv). 2000; 42(3):55-9.

43. Rose G. Sick individuals and sick populations. Int J Epidemiol. 1985;14(1):32-8.

44. Almeida C, Travassos C, Porto S, Labra ME. Health sector reform in Brazil: a case study of inequity. Int J Health Serv. 2000;30(1):129-62.

45. Whitehead M. The concepts and principles of equity and health. Int J Health Serv. 1992; 22(3):429-45.
46. Buarque C. A revolução nas prioridades: da modernidade técnica à modernidade ética. São Paulo: Paz e Terra; 1994.

47. Narvai PC. Recursos humanos para a promoção da saúde bucal: um olhar no início do século XXI. Em: Kriger L, org. Promoção da saúde bucal. $2^{\mathrm{a}}$ ed. São Paulo: Aboprev-Artes Médicas; 2003. Pp. 449-63.

48. Nunes A, Santos JRS, Barata RB, Vianna SM. Medindo as desigualdades em saúde no Brasil: uma proposta de monitoramento. Brasília: Organização Pan-Americana da Saúde/ Instituto de Pesquisa Econômica Aplicada; 2001.

49. Barros AJD, Bertoldi AD. Inequalities in utilization and access to dental services: a nationwide assessment. Cienc Saude Coletiva. 2002; 7(4):709-17.

50. Brasil. Constituição da República Federativa do Brasil-1988. Brasília: Senado Federal; 1988.

51. Victora CG, Vaughan JP, Barros FC, Silva AC, Tomasi E. Explaining trends in inequities: evidence from Brazilian child health studies. Lancet. 2000;356(9235);1093-8.

52. $3^{\text {a }}$ Conferência Nacional de Saúde Bucal. Brasília, 29/7/04 a 1/8/04. Relatório Final. Brasília: Ministério da Saúde; 2005.

Manuscrito recebido em 18 de julho de 2005. Aceito em versão revisada em 17 de abril de 2006.

ABSTRACT Objective. To examine the caries situation in Brazilian schoolchildren between 1980 and 2003 and to determine the distribution of caries and access to treatment in this population group.

Dental caries in Brazil: Methods. We employed secondary data concerning the number of decayed, missing or filled teeth (DMFT). The studies whose data were used differed in terms of study type, study design, sampling methods, and diagnostic criteria, but yielded national estimates that are considered valid for the DMFT index. Therefore, a trend analysis based on these studies was thought to be feasible.

Results. Analysis of DMFT values revealed a high frequency of dental caries in 1980 and a moderate frequency in the 1990s. In 2003, the DMFT index was still within the moderate range $(2,8)$. Between 1980 and 2003, a 61.7\% decrease in DMFT frequency was observed. The percentage of children with no DMFT increased from $3.7 \%$ in 1986 to $31.1 \%$ in 2003 . On the other hand, in the segment of the study population least affected, the percentage of children who received care increased from 26.3 in 1986 to 34.7 in 2003, yet in the group with DMFT this percentage fell from 50.2 in 1986 to 39.3 in 2003. In the segment with DMFT $\geq 6$, the percentage of those who received care remained stable (28\%). During the study period, $60 \%$ of the dental caries were found in $20 \%$ of the study population.

Conclusion. An important decline in DMFT was observed between 1980 and 2003 , perhaps as a result of increased access to fluoridated water and toothpaste and of changes in the goals of public oral health programs. Despite the improvement, caries is unevenly distributed in the pediatric population; a small proportion of individuals carries most of the disease burden. In addition, the proportion of teeth with caries that went untreated did not change between 1980 and 2003. Reducing socioeconomic disparities and adopting public health measures that target and reach the most vulnerable groups remain a challenge for policy makers in Brazil.

Key words Toothpaste, equity in access, fluoridation, DMF index, Brazil. 